\title{
One night in Venus, a lifetime with Mercury
}

\author{
Author: Chin Pey Yap ${ }^{\mathrm{A}}$
}

\section{Introduction}

Syphilis is known as the great mimicker in medicine because its non-specific symptoms can resemble many other diseases. It can often be overlooked by clinicians who only see syphilis infrequently. Syphilis is easy to cure in its early stages, but if left untreated, people can be left with debilitating complications.

The disease should be considered in all patients with multiple unexplained clinical presentations.

\section{Materials and methods}

A 54-year-old man with non-simultaneous indeterminate symptoms was seen by several medical specialties. He had a battery of investigations, which did not explain his symptoms.

He had headaches, which were treated as possible giant cell arteritis and a florid maculopapular rash, which was diagnosed as drug-related rash. His tongue ulcer had an unremarkable biopsy.

Six months from the onset of his first systemic symptom, he developed a relative scotoma in his vision. Examination findings were consistent with acute syphilitic posterior placoid chorioretinitis (ASPPC). Subsequent serology testing confirmed the diagnosis of syphilis.

His collective symptoms were reviewed in light of his new systemic diagnosis.

\section{Results and discussion}

The patient was treated with steroids and anti-treponemal medications (procaine benzylpenicillin and probenecid).

Diagnosing the patient before the disease progresses to its later stage has given him a better prognostic outlook.

\section{Conclusion}

This case highlights the importance of having a low threshold for investigating for syphilis in patients with multiple unexplained symptoms, and extends the context of ASPPC as a rare manifestation of syphilis.
Concise medical history taking helps in pointing to the relevant investigations and earlier diagnosis. Optimal management of syphilis involves multidisciplinary team input.

\section{Conflicts of interest}

None declared. 